\title{
Paraplegia
}

\section{Shoulder Pain in Acute Traumatic Quadriplegia}

\author{
W. P. Waring, MS, MD, ${ }^{1,2}$ F. M. Maynard, $M^{1}$ \\ ${ }^{1}$ The University of Michigan, Department of Physical Medicine and Rehabilitation, \\ Ann Arbor, Michigan 48109, ${ }^{2}$ Rehabilitation Medicine Service, Veterans Administration \\ Medical Center, Ann Arbor, Michigan, 48105, USA.
}

\section{Summary}

Fifty two patients with traumatic quadriplegia admitted to a spinal cord injury program within 6 months of injury were studied retrospectively. Seventy five per cent had shoulder pain documented in their medical records during initial rehabilitation, and $60 \%$ had shoulder pain for 2 weeks or more. When shoulder pain was documented it was bilateral in $61 \%$ of the cases. Age greater than 50 years, decreased shoulder range of motion, and not receiving shoulder exercise during the first 2 weeks after injury were positive risk factors associated with the onset of shoulder pain. At discharge $42 \%$ of the patients with shoulder pain were pain-free, $35 \%$ were noted to have improvement of their pain, and $23 \%$ had the same or worse shoulder pain. Study results demonstrate the high incidence of shoulder pain during initial rehabilitation of patients with traumatic quadriplegia and the importance of starting shoulder exercises during early acute care.

Key words: Spinal cord injuries; Shoulder pain; Rehabilitation.

Pain in patients with traumatic spinal cord injuries (SCI's) is a well documented finding. (Donovan et al., 1982; Burke, 1973). Estimates have been made that up to $70 \%$ of patients with SCI have chronic pain. (Nepomuceno et al., 1979; Burke, 1982; Guttmann, 1976). Shoulder pain occurring in patients with SCI's is of special interest because of their dependence on their upper extremities for the basic activities of daily living such as wheelchair propulsion, transfers, and dressing. Shoulder pain may also be one of the most common musculoskeletal pain problems among people with SCI's. It can be acute or chronic, can be localised or diffuse, and can have many aetiologies. A 1979 survey from England reported that over $50 \%$ of paraplegics and quadriplegics have chronic shoulder pain. (Nichols et al., 
1979). Fleming and Dawson reported in 1959 that 14 of $18(78 \%)$ new patients with quadriplegia admitted for initial rehabilitation had shoulder or neck pain. (Fleming and Dawson, 1958). Beyond this one small series, there is little information concerning shoulder pain during the initial rehabilitation of patients with quadriplegia, although its frequent occurrence is often mentioned. A restrospective study of our experience with this problem was undertaken to describe the incidence of this problem and investigate possible risk factors for its occurrence.

\section{Methods}

The medical records of patients with acute traumatic quadriplegia admitted to the University of Michigan's Spinal Cord Injury Program during a 3-year period (1980-1983) were retrospectively reviewed for all notations about shoulder pain, specifically including entries by members of the multidisciplinary team of physicians, occupational and physical therapists, nurses, social workers, and psychologists. Evaluation of shoulder pain did not follow a study protocol, and Xrays were taken as clinically indicated. Neck pain, trapezius pain, and pain at the end range of motion of the shoulder were not considered to meet documentation criteria for shoulder pain. Patients were excluded if they were admitted greater than 6 months following onset of injury. On the basis of this review, patients were divided into: (1) those with shoulder pain, who had documentation of shoulder pain for at least 2 weeks or more and (2) those without shoulder pain, who had no documentation of shoulder pain or whose shoulder pain was of less than 2 weeks duration. Patients were assigned a neurological level of injury and severity of injury, using Frankel grades of incompleteness, according to the standards of the American Spinal Injury Association (ASIA, 1982). Risk ratios and their 95\% confidence intervals were computed using dEPID software (Sullivan and Foster, 1987).

\section{Results}

The medical records of 55 patients who met the study criteria were reviewed. Three patients were excluded from further analysis: 2 patients with a complete motor/sensory lesion above C4 with anaesthetic shoulders and 1 patient with dementia. The 52 remaining patients comprised our study group. Eighty one per cent were males and their mean age was $29 \cdot 3$ years. The aetiology of quadriplegia included 25 motor vehicle accidents; 17 diving accidents; 7 falls; and 3 other types of injuries. Thirty nine patients $(75 \%)$ had shoulder pain mentioned at least once in their medical records; 31 patients $(60 \%)$ had 2 weeks or more of shoulder pain documented and were placed in the group with shoulder pain.

Among the patients in the group with shoulder pain, 39\% (12) had unilateral shoulder pain while $61 \%$ (19) had bilateral pain. The onset of shoulder pain was within the first 3 days post-injury in $39 \%$ and within the first 2 weeks in $52 \%$. The remaining $48 \%$ had the onset of shoulder pain documented between 2 weeks and 4 months post-injury.

Sixty per cent $(15 / 25)$ of patients with quadriplegia caused by motor vehicle accidents were in the group with shoulder pain, as compared to $59 \%(16 / 27)$ of patients with other aetiologies of injury. Seventy two per cent $(16 / 22)$ of patients with C3, C4, or C5 neurological levels of injury were in the group with shoulder 
pain as compared to $50 \%$ of patients $(15 / 30)$ with lower cervical levels of injury (C6, C7, C8). Sixty per cent (21/35) of patients with surgical management of their cervical injuries were in the group with shoulder pain as compared to $59 \%(10 / 17)$ of patients with nonsurgical management. Sixty two per cent $(15 / 24)$ of patients in whom halo-vest cervical immobilisation was used were in the group with shoulder pain as compared to $55 \%(17 / 31)$ of patients in whom other methods of cervical immobilisation were used. All patients (5) over the age of 50 years were in the group with shoulder pain.

Forty nine patients (94\%) had clear documentation of when bedrest was ended. Forty six per cent $(6 / 13)$ of patients who achieved upright sitting in a wheelchair (60 degrees for at least 2 hours a day) within a month of injury were in the group with shoulder pain as compared to $64 \%(23 / 36)$ of patients who started to sit after 1 month of injury.

Thirty five patients had a known date when shoulder exercises were started. Eighty six per cent $(6 / 7)$ of patients who started formal shoulder range of motion exercises after the first 2 weeks post-injury were in the group with shoulder pain as compared to $54 \%$ (15/28) of patients who had range of motion exercises started within the first 2 weeks post-injury. Only $36 \%$ (10/28) of patients with normal shoulder range of motion throughout their hospitalisation were in the group with shoulder pain as compared to $87 \%(21 / 24)$ of patients who had decreased shoulder range of motion at some time during their hospitalisation. Of the 21 patients in the group with shoulder pain who had decreased shoulder range of motion, 12 had clear documentation of the sequence for the onset of pain and decreased range of motion; 6 had the onset of pain and decreased range of motion simultaneously, 5 had shoulder pain after shoulder range of motion decreased, and only 1 patient had pain before range of motion decreased.

Selected variables that were associated with inclusion in the group with shoulder pain are listed in Table I with their risk ratios and $95 \%$ confidence intervals. Table II lists the specific diagnoses that were documented in the medical records for the group with shoulder pain. When no diagnosis was made for the cause or type of shoulder pain it was considered of unknown aetiology. There were no cases with shoulder subluxation or heterotopic ossification of the shoulder girdle.

The patients who developed shoulder pain were treated with traditional therapeutic modalities including hot packs, ultrasound, joint injection, stretching, and range of motion exercise, as well as non-steroidal anti-inflammatory agents and/or analgesics.

Table I Risk ratios for development of shoulder pain

\begin{tabular}{lcc}
\hline & Risk ratio & $\begin{array}{c}95 \% \text { Confidence } \\
\text { interval }\end{array}$ \\
\hline Age greater than 50 years & 1.95 & $1 \cdot 35-2 \cdot 82$ \\
C5 or higher neurological level & 1.45 & $0.94-2 \cdot 26$ \\
Motor vehicle accident & 1.01 & $0.65-1.58$ \\
Cervical surgery & 1.02 & $0.63-1.65$ \\
Sitting after 1 month of injury & 1.38 & $0.73-2.62$ \\
Range of motion exercise after 2 weeks of injury & 1.60 & $1.01-2.53$ \\
Decreased shoulder range of motion & 2.45 & $1 \cdot 46-4 \cdot 12$ \\
\hline
\end{tabular}


Table II Specific diagnoses of group with shoulder pain

\begin{tabular}{lr}
\hline \multicolumn{1}{c}{ Diagnoses } & \\
\cline { 2 - 2 } Unknown & 15 \\
Tendonitis & 5 \\
'Musculoskeletal' & 4 \\
Capsulitis & 4 \\
Fracture & 4 \\
Radiculopathy & 2 \\
Reflex sympathetic dystrophy & 2 \\
Prior degenerative arthritis & 2 \\
Referred & 1 \\
Prior rotator cuff tear & 1 \\
\hline
\end{tabular}

At discharge among the patients in the group with shoulder pain, $42 \%$ had resolution of shoulder pain; $35 \%$ had improvement of their shoulder pain; and $23 \%$ had the same or worse shoulder pain. For the 21 patients with both shoulder pain and decreased range of motion, $29 \%$ had normal shoulder range of motion at discharge; $38 \%$ had their range of motion improved; and 33\% had the same or worsening shoulder range of motion. All the patients with the same or worsening shoulder range of motion at discharge had shoulder pain at discharge. Only 2 patients had developed spasticity in their shoulder muscles by discharge.

\section{Discussion}

This study provides documentation that shoulder pain is indeed a common problem among patients with acute quadriplegia during initial rehabilitation and that it is often bilateral and associated with a loss of normal shoulder range of motion. There are probably many inciting factors that set the stage of pain, inhibition of movement, and eventually loss of joint motion. Some of the contributing factors to the high incidence of shoulder pain in patients with acute quadriplegia include: (1) weakness of the shoulder girdle muscles, especially the degree of weakness that occurs with C6 or higher motor levels of injury, can severely limit active joint movement and lead to muscle shortening and shoulder capsule tightness. Weakness can also cause imbalance of the force coupling of the rotator cuff, deltoid, biceps, and trapezius muslces that keeps the humeral head depressed and in the glenoid fossa. (Donovan and Kraft, 1974). The higher incidence of shoulder pain in our subjects with higher cervical lesions supports this theory; (2) neuritic shoulder pain can occur from nerve root injury or accompany radicular pain with dyesthesias or phantom sensations. This can be a special problem in the shoulders of patients with quadriplegia since many of the cervical dermatomes cross the shoulder area and provide primary sensory innervation to the shoulder joint and its supporting soft tissues; (3) referred pain to the shoulder can follow established patterns and commonly accompanies concurrent neck and trapezius muscle pain. Neck pain is common since fractures and/or dislocations of the cervical spine occur in the majority of cases of traumatic quadriplegia. Trapezius muscle pain is also common in traumatic quadriplegia since this muscle assists with both neck and shoulder girdle movement. In cases with shoulder girdle weakness the trapezius 
muscle can be over-used since it may be the only functioning shoulder muscle providing scapular elevation and retraction. Another problem is when shoulder protractors and depressors do not provide sufficient opposition to the trapezius muscle to prevent its shortening: (4) shoulder pain and inhibition of shoulder motion can occur from direct trauma at the time of original spinal injury or during initial hospitalisation. There was not an increased incidence of shoulder pain in our patients involved in motor vehicle accidents who were more likely to have associated acute injuries to shoulder structures. Trauma to the shoulder joint may occur during the acute medical/surgical stabilisation period due to suboptimal extremity positioning and/or poor techniques of turning and transferring patients. These types of shoulder trauma may be undetected due to narcotic use and/or apparent or occult closed head injuries; (5) pre-existing shoulder dysfunction may have the potential to exacerbate pain problems of the shoulder after the onset of quadriplegia. This would be especially true when coupling degenerative diseases of the shoulder with the daily use of this joint for many activities of daily living with quadriplegia. The $100 \%$ incidence of shoulder pain in our patients over the age of 50 years supports this theory; and (6) psychological issues related to secondary gain, control, manipulation and premorbid mental health problems may exacerbate any pain problem. An understudied psychological factor could be the difficulty of dealing with pain when there is an occult brain injury. Recent studies have estimated that up to $50 \%$ of American spinal cord patients had an occult brain injury at the time of their spinal injury (Davidoff et al., 1988).

There is probably no single or predominant aetiology to explain all cases of shoulder pain seen in the initial rehabilitation setting, as illustrated by the lack of a clear diagnosis for many patients. In retrospect, we suspect the diagnosis of shoulder capsulitis was under-utilised. In view of the retrospective design of this study, the incidence of shoulder pain is probably underestimated because only more severe cases are likely to have had clear documentation.

Our findings clearly support the importance of early and consistent shoulder range of motion exercises. Over half of the patients with shoulder pain developed it within 2 weeks of their spinal injury and delay in initiation of shoulder range of motion exercises beyond 2 weeks post-injury was found to be a significant risk factor for shoulder pain. Although there was only a small number of patients (12) in whom the sequence of the onset of pain and loss of shoulder range of motion was clearly documented, the fact that in 11 cases the loss of shoulder motion occurred with or after the onset of pain illustrates the potential cycle of pain, inhibition of motion causing loss of motion, and more pain. Scott and Donovan have reported special positioning techniques to prevent loss of motion in a specialised SCI centre setting. They assert that prolonged positioning of the shoulder in 90 degrees of abduction, combined with other positioning techniques, decreases the frequency and severity of shoulder pain (Scott and Donovan, 1981).

Care must be taken not to extrapolate the findings of this study with acute injuries to the increasingly common reports of shoulder problems in long term survivors of traumatic myelopathies (PVA, 1988). There are distinct differences between patients in the initial rehabilitation setting and those seen for long term outpatient care. Patients in the initial rehabilitation hospitalisation setting are young, often spend long periods in bed, and have the potential for significant motor recovery in the first year post-injury. Patients with long term quadriplegia 
are at risk for other problems not commonly seen in the initial rehabilitation setting such as degenerative arthritis, rotator cuff tears, shoulder subluxation, and muscle shortening due to spasticity. Caution must also be exercised in comparing the shoulder pain of people with quadriplegia to people with paraplegia since different aetiologic factors may be present (Gellman et al., 1988; Blankstein et al., 1985; Aljure et al., 1985; Wylie and Chakera, 1988). Further research is needed to determine if shoulder pain during initial rehabilitation predisposes patients with quadriplegia to the development of chronic shoulder pain.

Four major conclusions can be made from this study. Firstly, starting shoulder range of motion exercises as early as possible after injury is important to minimise shoulder pain. Secondly, loss of shoulder range of motion is a common sequela of shoulder pain, if not one of the common causes. Thirdly, older patients and patients with higher cervical injuries are more at risk to develop shoulder pain. Lastly, most patients improved with both their shoulder pain and shoulder range of motion by the time of their discharge from initial rehabilitation.

\section{References}

Aljure J, Eltorai I, Bradley WE, Lin JE, Johnson B 1985 Carpal tunnel syndrome in paraplegic patients. Paraplegia 23:182-6.

American SPINAL INJURy Association (ASIA), 250 East superior Street, Room 619 Chicago, Illinois.

Blankstein A, Shmueli R, Weingarten I, Engel J, Ohry A 1985 Hand problems due to prolonged use of crutches and wheelchairs. Orthopedic Review 14:29-34.

BURKE DC 1982 Pain and phantom sensation-a neglected problem of spinal paralysis. Paper presented at Third Far East and South Pacific Spinal Conference.

BURKE DC 1973 Pain in paraplegia. Paraplegia 10:297-313.

Davidoff G, Thomas P, Johnson M, Berent S, Dijkers M, Doljanac R 1988 Closed head injury in acute traumatic spinal cord injury: incidence and risk factors. Archives of Physical Medicine and Rehabilitation 69:869-872.

Donovan WH, Dimitrijevic MR, Dahm L, Dimitrijevic M 1982 Neurophysiological approaches to chronic pain following spinal cord injury. Paraplegia 20:135-146.

DONOVAN WH, KRAFT GH 1974 Rotator cuff tear versus suprascapular nerve injury. Archives of Physical Medicine and Rehabilitation 55:424-428.

Fleming W, DAwSON AR 1958 Shoulder pain in quadriplegic patient: a theory as to its cause. Southern Medical fournal 51:1460-1463.

GeLLMAN J, IEN S, WATER RL 1988 Late complications of the weight-bearing upper extremity in the paraplegic patient. Clinical Orthopedics and Related Research 233:132-135.

GUTTMANN L 1976 Spinal Cord Injuries. London: Adlard and Son, 281-287.

NePOMUCENo C, FINE PR, RichardS JS et al., 1979 Pain in patients with spinal cord injury. Archives of Physical Medicine and Rehabilitation 60:605-608.

Nichols PJR, NoRman PA, ENNIS JR 1979 Wheelchair users' shoulder? Scandinavian fournal of Rehabilitation Medicine 11:29-32.

PaRAlyZed VETERANS OF AMERICA 1988 PVA needs assessment questionnaire: Draft report.

SCOTT J, DONOVAN W 1981 The prevention of shoulder pain and contracture in the acute tetraplegic patient. Paraplegia 19:313-319.

SUlLIVAN KM, FOSTER DA 1987 dEPID: A program for stratified and standardized analysis. Version 2.1, Division of Nutrition, Centers for Disease Control, 1660 Clifton Road, Atlanta, Georgia.

WYLIE EJ, CHAKERA TMH 1988 Degenerative joint abnormalities in patients with paraplegia of duration greater than 20 years. Paraplegia 26:101-106. 